\title{
The Northern $\chi$-Orionid meteoroid stream and possible association with the potentially hazardous asteroid 2008XM1
}

\author{
José M. Madiedo, ${ }^{1,2 \star}$ Josep M. Trigo-Rodríguez, ${ }^{3}$ Iwan P. Williams, ${ }^{4}$ José L. Ortiz ${ }^{5}$ \\ and Jesús Cabrera ${ }^{2}$ \\ ${ }^{1}$ Facultad de Ciencias Experimentales, Universidad de Huelva, 21071 Huelva, Spain \\ ${ }^{2}$ Departamento de Física Atómica, Molecular y Nuclear, Facultad de Física, Universidad de Sevilla, 41012 Sevilla, Spain \\ ${ }^{3}$ Institute of Space Sciences (CSIC-IEEC), Campus UAB, Facultat de Ciències, Torre C5-parell-2a , 08193 Bellaterra, Barcelona, Spain \\ ${ }^{4}$ School of Physics and Astronomy, Queen Mary, University of London, London, UK \\ ${ }^{5}$ Instituto de Astrofísica de Andalucía, CSIC, Apt. 3004, Camino Bajo de Huetor 50, 18080 Granada, Spain
}

Accepted 2013 February 21. Received 2013 February 8; in original form 2012 December 13

\begin{abstract}
We present new orbital data and dynamic results pointing towards the origin of the Northern $\chi$-Orionid meteoroid stream, which is a part of the Taurid meteoroid complex. A new software package was developed to establish the potential parent bodies of meteoroid streams based on the similarity of their orbits. The analysis of a Northern $\chi$-Orionid fireball observed on 2011 December 6 identified two potential parent bodies: the near-Earth object (NEO) 2002XM35 (previously proposed as the parent of this meteoroid stream) and the more recently discovered potentially hazardous asteroid 2008XM1. The calculation of the evolution of the orbital elements performed by using the Mercury 6 symplectic integrator supports the idea that 2008XM1 is a better parent body. Our data sample was expanded by including also in the calculations the mean orbit of the $\chi$-Orionid stream. The results are consistent with the fragmentation of a larger body in the past that could give rise to both NEOs and the Northern $\chi$-Orionid stream. To confirm this, further observations to improve the orbital elements of these asteroids should be attempted before the objects are lost. The analysis of the emission spectrum recorded for this fireball supports a primitive nature for these meteoroids.
\end{abstract}

Key words: meteorites, meteors, meteoroids-minor planets, asteroids: individual: 2002XM35 - minor planets, asteroids: individual: 2008XM1.

\section{INTRODUCTION}

Most of the major meteoroid streams have well-defined parent bodies, but the origin of the vast majority of the minor streams remains unknown (Jenniskens 2006). There are several reasons for this; for example, minor streams have usually a very few meteors whose orbits have been well determined, leading to large uncertainty in the mean orbital elements of the stream. In many cases the parent of a minor stream may also be small, so that detection is difficult and a good orbit might not be known. The main reason however is probably the relatively rapid orbital evolution of both meteoroid streams and their parent bodies in the near-Earth region (Hughes, Williams \& Fox 1981; Jenniskens 1998). The identification of the progenitor bodies of meteoroid streams is crucial because it increases our knowledge on the origin and evolution of meteoroid streams, but more important a study of the meteors allows us to make valuable deductions about chemical and physical properties of the parent and the meteoroid ejection process or break-up of the parent. These associations can also provide important clues about potential meteorite-dropping fireball events with an origin in the near-Earth object (NEO) population, and identify potential current sources of primitive meteorites (Trigo-Rodríguez et al. 2007a, 2009).

Ever since Schiaparelli (1867) identified the pairing between the Perseid stream and comet 109P/Swift-Tuttle, similarity of the orbits has been the main tool used for establishing a connection between a meteoroid stream and its parent [see Williams (2011) for a historical account of this topic]. A number of authors have proposed criteria for quantifying the difference between two orbits, the first being by Southworth \& Hawkins (1963). For two orbits A and B, they defined $D_{\mathrm{SH}}$ by

$$
\begin{aligned}
D_{\mathrm{SH}}^{2}= & \left(e_{B}-e_{A}\right)^{2}+\left(q_{B}-q_{A}\right)^{2}+\left(2 \sin \frac{I_{B A}}{2}\right)^{2} \\
& +\left(\frac{e_{A}+e_{B}}{2}\right)^{2}\left(2 \sin \frac{\pi_{B A}}{2}\right)^{2}
\end{aligned}
$$

^E-mail: madiedo@cica.es 
where $e$ and $q$ are, respectively, the eccentricity and perihelion distance in astronomical units, $I_{B A}$ is the angle between the two orbital planes and $\pi_{B A}$ is the difference between the longitudes of perihelia as measured from the intersection of both orbits. A small value of $D_{\mathrm{SH}}$ implies a small difference so that the two orbits can be regarded as similar. Usually $D_{\mathrm{SH}}<0.15$ is adopted (Lindblad 1971a,b). A simplified $D$ criterion where the dependence on the two angles $\omega$ and $\Omega$ is dropped was proposed by Steel, Asher \& Clube (1991) as both these angles evolve much faster than the other three orbital elements.

Other criteria have been proposed by Drummond (1981), Jopek (1993), Valsecchi, Jopek \& Froeschlé (1999), Jopek, Rudawska \& Bartczak (2008), Nesvorny \& Vokrouhlicky (2006) and Jenniskens (2008). This last study defines

$$
D_{\mathrm{J}}^{2}=\left(\frac{C_{A 1}-C_{B 1}}{0.13}\right)^{2}+\left(\frac{C_{A 2}-C_{B 2}}{0.06}\right)^{2}+\left(\frac{C_{A 3}-C_{B 3}}{14.2^{\circ}}\right)^{2}
$$

with

$$
\begin{aligned}
& C_{1}=(1-e)^{2} \cos ^{2} i \\
& C_{2}=e^{2}\left(0.4-\sin ^{2} i \sin ^{2} \omega\right) \\
& C_{3}=\omega+\Omega .
\end{aligned}
$$

For the $D_{\text {J }}$ criterion, an upper threshold value of 0.879 has been proposed by Rudawska, Vaubaillon \& Atreya (2012). This value was suggested on the basis of simulations performed on artificial data sets of four meteor streams for which the parent body is known (namely the Leonids, Perseids, Draconids and $\tau$-Herculids). However, higher $D_{\mathrm{J}}$ values (between 0.90 and 1.49) have been found when comparing the orbit of some meteor showers and their wellestablished parent bodies [see for instance table 1 in Jenniskens (2008)]. In fact, according to Jenniskens (2008), the condition $D_{\mathrm{J}}<$ 1.5 would be satisfied by objects related through earlier fragmentation events.

Based on orbital similarity, many authors have suggested associations between near-Earth asteroids and meteoroid streams. Most of the associations have involved relatively minor showers and a list of some potential pairings is given in table 9 of Jenniskens (2006). The systematic monitoring of the skies, principally to identify asteroids that may present a danger to Earth, has led to a vast increase in the number of known NEOs. This has led to a corresponding increase in the number of claimed associations between meteoroid streams and asteroids. Babadzhanov, Williams \& Kokhirova (2008) calculated that the probability of any two orbits in the near-Earth environment having a $D$ value less than 0.3 was nearly 20 per cent, so that there is a 1 in 5 chance that any given calculated pair is spurious.

Porubčan, Williams \& Kornoš (2004) suggested that similarity of orbits should be maintained for a $5000 \mathrm{yr}$ period before a generic association should be claimed, a view later supported by TrigoRodríguez et al. (2007a). We have developed a software package to establish meteoroid-parent body dynamic associations on the basis of continuous computation of dissimilarity criteria over time. ORbital Association Software (ORAS) is a software developed within

Table 1. Geographical coordinates of the meteor observing stations involved in this work.

\begin{tabular}{lcccc}
\hline Station no. & Station name & Longitude (W) & Latitude (N) & Alt. (m) \\
\hline 1 & Sevilla & $05^{\circ} 58^{\prime} 50^{\prime \prime}$ & $37^{\circ} 20^{\prime} 46^{\prime \prime}$ & 28 \\
2 & El Arenosillo & $06^{\circ} 43^{\prime} 58^{\prime \prime}$ & $37^{\circ} 06^{\prime} 16^{\prime \prime}$ & 40 \\
\hline
\end{tabular}

the SPanish Meteor Network (SPMN) project to identify new minor bodies presumably associated with meteoroid streams (Madiedo et al. 2011c).

In order to establish a relationship between the orbit of the meteoroids and that of their potential parent bodies, the availability of precise orbital parameters is fundamental. We have employed here our orbital association software tool to analyse the likely origin of the Northern $\chi$-Orionids. The $\chi$-Orionid shower is a part of the Taurid complex, a complex known to consist of a large number of minor meteor streams and substreams (Denning 1928) and has northern (ORN) and southern (ORS) branches identified by Whipple (1940). A list of these can be found in Porubčan, Kornoš \& Williams (2006) or Jenniskens (2006). The activity period of the Northern $\chi$ Orionids extends from December 1 to January 5, with a maximum on December 11. The Southern $\chi$-Orionids are active from about December 1 to January 16, with a maximum also on December 12 (Jenniskens 2006). Additional data about these meteoroid streams can be obtained by attaching diffraction gratings to high-sensitivity CCD video cameras in order to obtain not only trajectory, radiant and orbital information but also clues on the chemical composition of the meteoroids (Trigo-Rodríguez et al. 2009). On 2011 December 6 , a mag. -7 double-station Northern $\chi$-Orionids fireball was observed by the SPMN. Its emission spectrum was also recorded. The analysis of this event, including its atmospheric trajectory, radiant, orbital parameters and chemical information, is described later in this paper.

In Porubčan et al. (2004, 2006) NEO 2002XM35 was proposed as the parent body of this low-inclination stream according to a comparison with the orbit of the Northern $\chi$-Orionids based on seven meteors. We have expanded our data sample by using the mean orbit of this stream based on 12 meteors (Jenniskens 2006). Using the new orbit for the Northern $\chi$-Orionids and searching through the orbit data base for asteroids discovered since 2004, we propose that the potentially hazardous asteroid (PHA) 2008XM1 is a better candidate as parent body.

\section{INSTRUMENTATION AND METHODS}

The two SPMN observing stations involved in the recording of the fireball analysed here (table 1) employ high-sensitivity Watec CCD video cameras (models $902 \mathrm{H}$ and $902 \mathrm{H}$ Ultimate from Watec Corporation, Japan) to monitor the night sky. A more detailed description of these systems and their operation has been given in Trigo-Rodríguez et al. (2007b) and Madiedo \& Trigo-Rodríguez (2007, 2010). Both stations perform continuous fireball monitoring and spectroscopic campaigns and work in an autonomous way by means of own software (Madiedo \& Trigo-Rodríguez 2010; Madiedo et al. 2010). For meteor spectroscopy we employ transmission diffraction gratings (500 or 1000 lines $\mathrm{mm}^{-1}$, depending on the device).

The first step in the data reduction process requires the identification of meteor trails simultaneously recorded from at least two different observing stations. This is automatically done by means of a software package, which searches through the data base of meteors that appeared during the same observing interval and in the appropriate position. Thus, this software checks if a given meteor has also been detected by another camera covering a common atmospheric volume from a different observing station. An astrometric measurement is then performed by hand in order to obtain the plate $(x, y)$ coordinates of the meteor along its apparent path from each station. Our AmaltheA software (Trigo-Rodríguez et al. 2009; Madiedo, Trigo-Rodríguez \& Lyytinen 2011a) transforms the 
astrometric plate coordinates into equatorial coordinates by using the position of reference stars appearing in the images. This package employs the method of the intersection of planes to determine the radiant and reconstruct the trajectory in the atmosphere of meteors recorded from at least two different observing stations (Ceplecha 1987). From the sequential measurements of the video frames and the trajectory length, the velocity of the meteor along its path is obtained. For the bolide discussed here, the pre-atmospheric velocity $V_{\infty}$ was found by fitting the velocities measured at the earliest part of the meteor trajectory, where the deceleration is negligible, to a linear model. In this way, $V_{\infty}$ was taken as the extrapolated value for a height of about $120 \mathrm{~km}$ above the ground level. Once this velocity and the atmospheric trajectory are known, the software computes the orbital parameters of the corresponding meteoroid by following the procedure described in Ceplecha (1987).

\section{OBSERVATIONS: TRAJECTORY, RADIANT AND ORBIT}

As part of our continuous meteor and fireball monitoring campaign, a Northern $\chi$-Orionid bolide (SPMN code 061211) was simultaneously recorded from two observing stations located in Andalusia (table 1) on 2011 December 6, at $20^{\mathrm{h}} 32^{\mathrm{m}} 59.4 \pm 0.1 \mathrm{~s}$ UTC (Fig. 1). Its emission spectrum was imaged from station 2 (Fig. 1b). As can be seen in Fig. 1 a, the final part of the fireball's trajectory could not be imaged from station 1 .

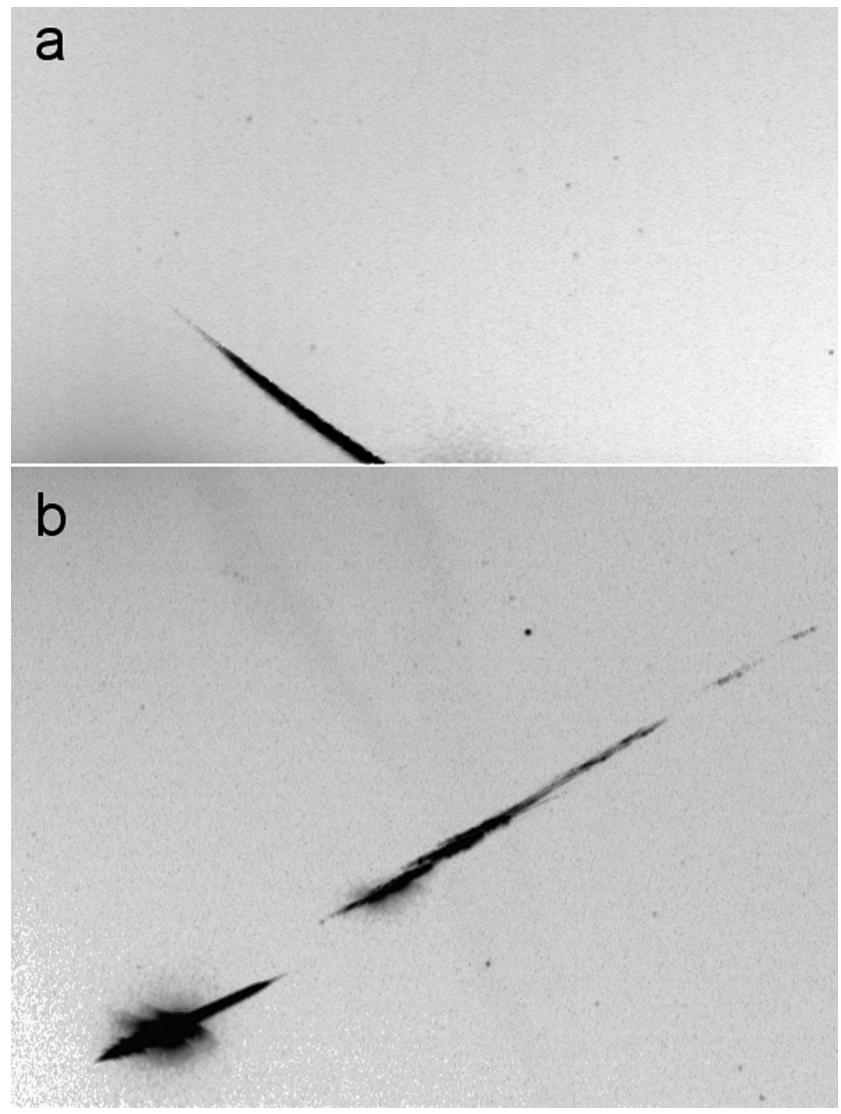

Figure 1. Composite negative image of the mag. $-7 \pm 1$ SPMN061211 Northern $\chi$-Orionid fireball, recorded on 2011 December 6 at $20^{\mathrm{h}} 32^{\mathrm{m}} 59.4 \pm 0.1 \mathrm{~s}$ UTC from (a) Sevilla and (b) El Arenosillo meteor observing stations. This latter image includes its emission spectrum, with the bolide (zero-order spectrum) on the lower-left part of the image.
Table 2. Trajectory and radiant data for the SPMN061211 Northern $\chi$-Orionid fireball (J2000).

\begin{tabular}{ccccccc}
\hline $\begin{array}{c}H_{\mathrm{b}} \\
(\mathrm{km})\end{array}$ & $\begin{array}{c}H_{\mathrm{e}} \\
(\mathrm{km})\end{array}$ & $\begin{array}{c}\alpha_{\mathrm{g}} \\
\left({ }^{\circ}\right)\end{array}$ & $\begin{array}{c}\delta_{\mathrm{g}} \\
\left({ }^{\circ}\right)\end{array}$ & $\begin{array}{c}V_{\infty} \\
\left(\mathrm{km} \mathrm{s}^{-1}\right)\end{array}$ & $\begin{array}{c}V_{\mathrm{g}} \\
\left(\mathrm{km} \mathrm{s}^{-1}\right)\end{array}$ & $\begin{array}{c}V_{\mathrm{h}} \\
\left(\mathrm{km} \mathrm{s}^{-1}\right)\end{array}$ \\
\hline 98.7 & 55.0 & $79.3 \pm 0.3$ & $26.5 \pm 0.2$ & $28.0 \pm 0.3$ & $25.4 \pm 0.3$ & $37.4 \pm 0.3$ \\
\hline
\end{tabular}

The magnitude of this fireball has been estimated by direct comparison of the brightness level of the pixels near the maximum luminosity of the meteor trail and those of nearby stars. Though the event was not particularly bright (absolute magnitude $=-7 \pm 1$ ), the alignment of the meteor trail with the Northern $\chi$-Orionids radiant attracted our attention. In order to confirm its association with this meteoroid stream, the atmospheric trajectory and radiant were derived. The fireball started its luminous path at a height $H_{\mathrm{b}}$ of $98.7 \pm 0.5 \mathrm{~km}$, with the terminal point of the trajectory at a height $H_{\mathrm{e}}=55.0 \pm 0.5 \mathrm{~km}$ above the ground level. The fireball experienced a bright flare probably associated with its catastrophic disruption at a height of $71.3 \pm 1.0 \mathrm{~km}$ when its velocity was $26.2 \pm 0.4 \mathrm{~km} \mathrm{~s}^{-1}$, from which a dynamic strength of $(4.2 \pm 0.3) \times 10^{4} \mathrm{dyn} \mathrm{cm}^{-2}$ was derived by using the procedure described in Trigo-Rodríguez \& Llorca (2006). The pre-atmospheric velocity, obtained as indicated above, was $V_{\infty}=28.0 \pm 0.3 \mathrm{~km} \mathrm{~s}^{-1}$. The apparent radiant was located at $\alpha=77.6 \pm 0.3, \delta=27.3 \pm 0.2$. The radiant and orbital parameters (J2000) are summarized in Tables 2 and 3, respectively, and these confirm that the fireball belongs to the Northern $\chi$-Orionid shower.

\section{DISCUSSION}

\subsection{Analysis of the Northern $\chi$-Orionid fireball spectrum}

The emission spectrum produced by the SPMN061211 Northern $\chi$-Orionid fireball was obtained by one of the spectral cameras operating from El Arenosillo (Fig. 1b). Despite its low resolution (about $1.2 \mathrm{~nm} \mathrm{pixel}^{-1}$ ), this spectrum provides an insight into the chemical nature of the meteoroid. We have followed the reduction procedure described in Trigo-Rodríguez et al. (2003) to analyse the spectrum and the calculations have been performed with our CHIMET software (Madiedo, Toscano \& Trigo-Rodríguez 2011b). The video frames containing the emission spectrum were darkframe subtracted and flat-fielded. The signal from different frames was then integrated and calibrated in wavelengths by identifying typical multiplets appearing in meteor spectra. Next, the intensity of the emission lines was corrected by using the spectral sensitivity curve of the recording device. The result is shown in Fig. 2. As can be seen, the contribution from ionized calcium $\mathrm{H}$ and $\mathrm{K}$ lines in the ultraviolet is blended with the emission from $\mathrm{Fe}_{\mathrm{I}}-4$ multiplet. Other noticeable emission lines correspond to Ca I-1 (422.7 nm), Fe I-41 $(440.4 \mathrm{~nm}), \mathrm{Mg} \mathrm{I}-2(517.3 \mathrm{~nm})$ and $\mathrm{Na} \mathrm{I}-1(588.9 \mathrm{~nm})$. Atmospheric $\mathrm{N}_{2}$ bands were also identified in the red region of the spectrum. Because of the low geocentric velocity of the meteoroid, the second (high-temperature) component in the spectrum will be almost nonexistent (Trigo-Rodríguez et al. 2003). Hence, the lines identified belong to the main component. Relative abundances of the main rocky elements in the meteor column are obtained with a software application to reconstruct a synthetic spectrum that enabled to adjust temperature $(T)$, the column density of atoms $(N)$, the damping constant $(D)$ and the surface area $(P)$ from the observed brightness of lines as explained in Trigo-Rodríguez et al. (2003). Then, the modelled spectrum of the meteor column is directly compared with 
Table 3. Orbital parameters (J2000) for the SPMN061211 Northern $\chi$-Orionid fireball, averaged orbit for the Northern $\chi$-Orionid (ORN) meteoroid stream (Jenniskens 2006), and orbital data for the two NEOs considered in this paper.

\begin{tabular}{lccccc}
\hline \multicolumn{1}{c}{ Object } & $a(\mathrm{au})$ & $e$ & $i\left(^{\circ}\right)$ & $\omega\left(^{\circ}\right)$ & $\Omega\left(^{\circ}\right)$ \\
\hline SPMN061211 & $2.2 \pm 0.1$ & $0.79 \pm 0.01$ & $3.2 \pm 0.6$ & $281.5 \pm 0.2$ & $254.2322 \pm 10^{-4}$ \\
ORN $(N=12)$ & 2.22 & 0.797 & 2.5 & 283.9 & 257.3 \\
2002XM35 & 2.3304 & 0.8361 & 3.0845 & 313.4382 & 229.2701 \\
2008XM1 & 2.3679 & 0.7822 & 4.9954 & 276.1411 & 259.8564 \\
\hline
\end{tabular}

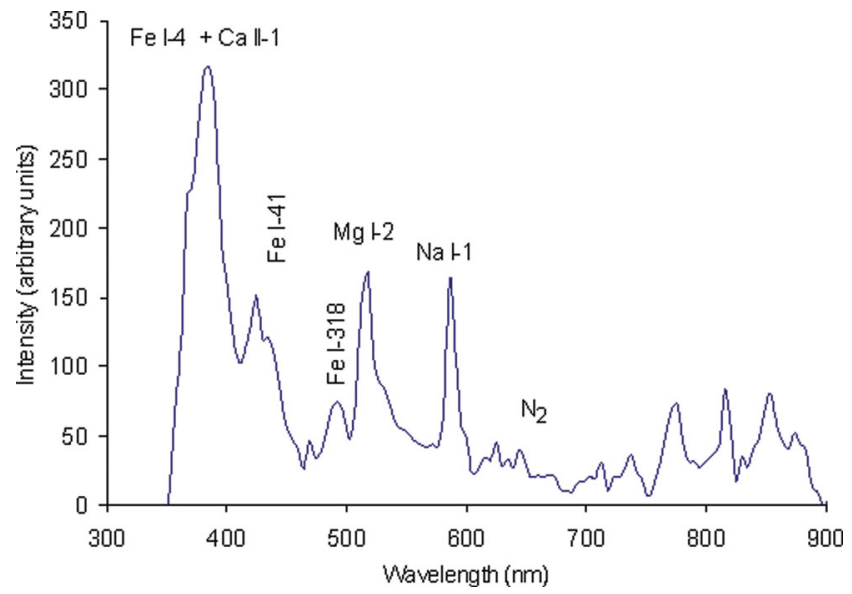

Figure 2. Calibrated emission spectrum of the SPMN061211 Northern $\chi$-Orionid fireball.

the observed one to fit all its peculiarities. In order to get the physical parameters in the meteor column, the intensity of the Fe I lines was fitted to the Fe multiplets previously identified in the spectrum. The values of the averaged meteor plasma temperature and $\mathrm{Fe}$ I column density were, respectively, $4900 \pm 200 \mathrm{~K}$ and $10^{14} \mathrm{~cm}^{-2}$. Once the three physical parameters previously mentioned $(T, D$ and $P$ ) are fixed, the relative spectrum abundances of the main rocky elements can be modified in successive iterative steps until obtaining an optimal fit. The relative elemental abundances that we provide here are those obtained as a consequence of such a process. By assuming an abundance ratio of $\mathrm{Fe} / \mathrm{Si}=1.16$ (Anders \& Grevesse 1989), the calculated relative abundances referred to $\mathrm{Si}$ were: $\mathrm{Mg} / \mathrm{Si}=0.60 \pm$ $0.01, \mathrm{Na} / \mathrm{Si}=0.035 \pm 0.001, \mathrm{Ca} / \mathrm{Si}=0.03 \pm 0.01, \mathrm{Cr} / \mathrm{Si}=0.02 \pm$ $0.01, \mathrm{Ni} / \mathrm{Si}=0.01 \pm 0.01, \mathrm{Ti} / \mathrm{Si}=0.002 \pm 0.001$ and $\mathrm{Al} / \mathrm{Si}=$ $0.07 \pm 0.01$. This assumption is not problematic due to the fact that, as we previously explained, the derived elemental abundances are relative to $\mathrm{Fe}$ obtained by direct comparison between the observed and the synthetic spectrum. Consequently, the final computed value e.g. $\mathrm{Na} / \mathrm{Fe}$ is multiplied by $\mathrm{Fe} / \mathrm{Si}=1.16$ to obtain directly the presumably chondritic $\mathrm{Na} / \mathrm{Si}$ ratio. We can directly compare the de- rived chemical abundances relative to $\mathrm{Si}$ with those obtained from other undifferentiated Solar system bodies (Table 4). We remark the measured underabundance of $\mathrm{Mg}$, which directly places the Northern $\chi$-Orionid materials more comparable with interplanetary dust particles (IDPs) or the measured GIOTTO 1P/Halley abundances than with $\mathrm{CI}$ and $\mathrm{CM}$ carbonaceous chondrites. Consequently, this meteoroid stream could have mm-sized meteoroids exhibiting an overabundant dark matrix, perhaps ultracarbonaceous in nature as previously found in collected Antarctic micrometeorites, and with a minor content in mineral grains than carbonaceous chondrites (Duprat et al. 2010).

\subsection{Orbital analysis: parent body searches}

The orbital data obtained for the SPMN061211 fireball can be used to infer information about the likely origin of the meteoroid. For this purpose, we have employed our ORAs program. By entering the orbital data of a given meteor, or an averaged orbit for a set of members of a stream, the software can search through several data bases in order to establish a potential link with other bodies in the Solar system. Thus, the current version of ORAs can use the NeoDys and Minor Planet Center data bases for the currently listed asteroids and comets known. The value of the semimajor axis, eccentricity, inclination, longitude of the ascending node and argument of the perihelion must be provided. Then, the user specifies which data base containing information about small bodies in the Solar system will be used and which dissimilarity criterion will be employed. With respect to the dissimilarity criterion, five choices are also available, namely those of Southworth \& Hawkins (1963), (Valsecci, Jopek \& Froeschlé 1999), Drummond (1981), Jopek (1993) and Jenniskens (2008). The cut-off value for the corresponding criterion must also be entered. All bodies that have criterion value lower than this cut-off value are listed by name and the corresponding value of the criteria displayed. The orbital parameters of a body that is not included in any of these data bases can also be inserted.

By using the Southworth \& Hawkings criterion for the SPMN061211 fireball, two candidates were obtained. One was NEO 2002XM35 $\left(D_{\mathrm{SH}}=0.14, D_{\mathrm{J}}=0.87\right)$, which has already been proposed as the potential parent body of the Northern $\chi$-Orionid

Table 4. Computed elemental abundances relative to Si of the Northern $\chi$-Orionid meteoroid (SPMN061211) compared with the inferred for other solar system undifferentiated materials (Rietmeijer 2000; Rietmeijer 2002a,b; Trigo-Rodríguez 2002, and references therein).

\begin{tabular}{lccccccc}
\hline Undifferentiated material & $\mathrm{Mg}$ & $\mathrm{Na}$ & $\mathrm{Ca}$ & $\mathrm{Cr}$ & $\mathrm{Ni}$ & $\mathrm{Ti}$ & $\mathrm{Al}$ \\
\hline SPMN061211 & 0.60 & 0.035 & 0.03 & 0.02 & 0.01 & 0.002 & 0.07 \\
1P/Halley & 0.54 & 0.054 & 0.034 & 0.050 & 0.22 & 0.002 & 0.037 \\
IDPs & 0.85 & 0.085 & 0.048 & 0.012 & 0.037 & 0.002 & 0.075 \\
CI chondrites & 1.06 & 0.060 & 0.071 & 0.013 & 0.051 & 0.002 & 0.085 \\
CM chondrites & 1.04 & 0.035 & 0.072 & 0.012 & 0.046 & 0.002 & 0.084 \\
\hline
\end{tabular}


meteoroid stream (Porubčan et al. 2004, 2006). The other was the PHA 2008XM1 $\left(D_{\mathrm{SH}}=0.05, D_{\mathrm{J}}=0.17\right)$. As can be seen, both the criteria have lower values for this latter body, showing that its orbit is much closer to that of the fireball. A numerical integration of the orbital parameters backwards in time has been performed in order to test the link between the SPMN061211 fireball and these two NEOs. To increase our data sample, the mean orbit of this stream obtained by Jenniskens (2006) from $12 \chi$-Orionid meteors was also included in the computations. The integrations were performed using the MERCURY 6 software (Chambers 1999). The gravitational fields of Venus, the Earth-Moon system, Mars, Jupiter and Saturn were considered. The orbits were integrated back for $5000 \mathrm{yr}$. For the Northern $\chi$-Orionid mean orbit, we considered the evolution with time of the orbital parameters of 18 theoretical meteoroids uniformly distributed in mean anomaly. The results are shown in Fig. 3, where the evolution of the semimajor axis, eccentricity, orbital inclination and argument of the perihelion is displayed. The grey areas in these plots correspond to the evolution of the 18 meteoroids representing the average Northern $\chi$-Orionid orbit. The evolution of these meteoroids and the orbit inferred from the SPMN061211 fireball is similar to the evolution of 2008XM1. As can be noticed, the worst match is obtained for the evolution of the orbital inclination. Fig. 4 shows that the evolution of the $D_{\mathrm{SH}}$ criterion reveals a link between the SPMN061211 fireball and NEO 2008XM1, with the values of $D_{\mathrm{SH}}$ being less than 0.15 over a time-scale of about $2600 \mathrm{yr}$. However, for the mean Northern $\chi$-Orionid orbit an association with 2002XM35 is favoured, although for 2008XM1 $D_{\mathrm{SH}}$ remains less than 0.23 for over $2800 \mathrm{yr}$.

When the $D_{\mathrm{J}}$ criterion is employed as is shown in Fig. 5, both the SPMN061211 fireball orbit and the average orbit of the Northern $\chi$-Orionid stream provide lower values when compared to the orbit of 2008XM1 rather than 2002XM35. For 2008XM1, the condition $D_{\mathrm{J}} \leq 0.87$ is satisfied during a time-scale of $5000 \mathrm{yr}$. However, for 2002XM35 divergences appear for times longer than about $3000 \mathrm{yr}$ when its orbit is compared to the average orbit of the Northern $\chi$-Orionids, but much earlier (about $500 \mathrm{yr}$ in the past) when this comparison is made with the SPMN061211 fireball.

As Fig. 6 shows, the $D_{\mathrm{SH}}$ criterion for both asteroids remains below 0.4 over a time-scale of $4000 \mathrm{yr}$. The $D_{\mathrm{J}}$ criterion calculated for these objects, however, predicts a better association for these NEOs (Fig. 7), with $D_{\text {J }}$ values below 1.24 over 5000 yr. This implies that these objects could be dynamically related (Jenniskens 2008) and opens the possibility that both asteroids may in some way be involved in the formation of the Northern $\chi$-Orionid meteoroid stream. A common possible scenario is a disruptive event taking place on a larger progenitor body that, at the same time, produced the Northern $\chi$-Orionid meteoroid stream. Alternatively, since $2008 \mathrm{XM} 1$ is slightly larger than $2002 \mathrm{XM} 35$, it may be that 2002XM35 should be regarded as a fragment that split off from 2008XM1 at the time of the stream formation.

Unfortunately however we must point out that the orbit of NEO 2002XM35 was determined on the basis of nine observations performed during just one day in 2002 and the object has not been observed since. Similarly, 2008XM1 is a one opposition object, though in this case 89 observations were obtained during a period of $9 \mathrm{~d}$ in 2008. The orbits of both objects must thus be regarded as uncertain and additional observations of both objects are urgently needed before both of these are lost for ever.

If either object were observed further by telescope, additional clues about relationships with the Northern $\chi$-Orionid stream could be also obtained, for instance, by obtaining the reflection spectra of these NEOs, so that their chemical properties could be correlated
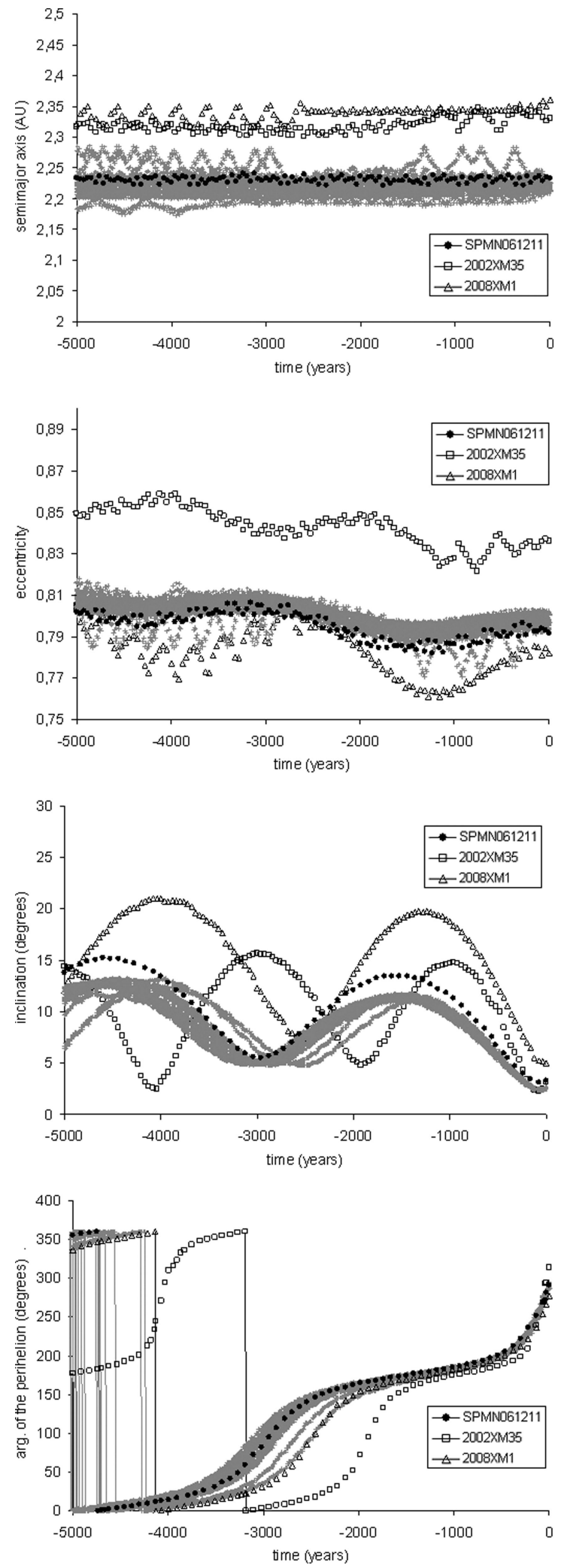

Figure 3. Numerical integration, over a time-scale of $5000 \mathrm{yr}$ since present time, of the evolution of the semimajor axis, eccentricity, inclination and longitude of the perihelion of the SPMN061211 bolide and both NEOs (2002XM35 and 2008XM1). The plots in grey correspond to the evolution of the orbit of 18 theoretical meteoroids uniformly distributed in mean anomaly about the mean Northern $\chi$-Orionid orbit. 


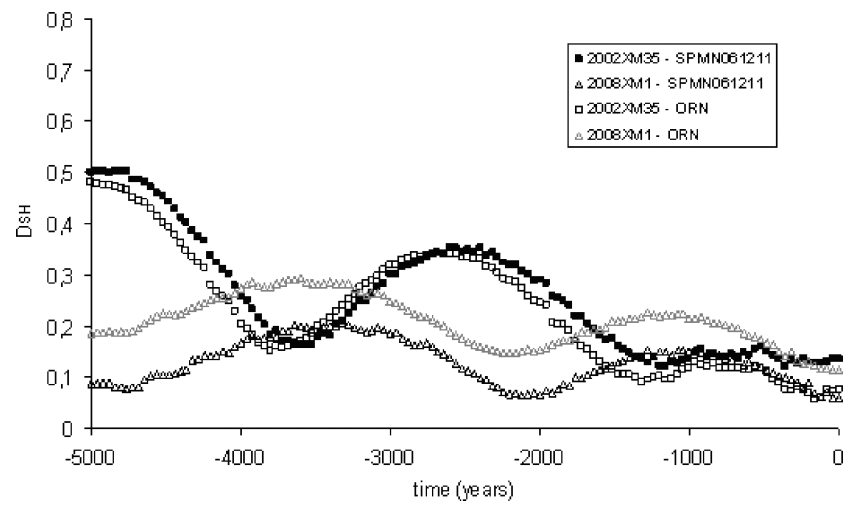

Figure 4. Evolution, as a function of time, of the $D_{\mathrm{SH}}$ criterion calculated by comparing the orbits of the Northern $\chi$-Orionids (ORN) and the SPMN061211 fireball with those of NEOs 2002XM35 and 2008XM1.

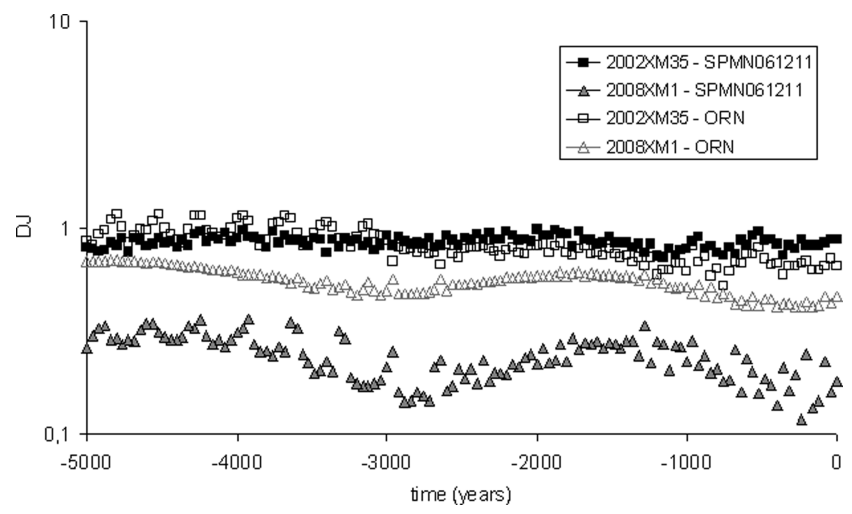

Figure 5. Evolution, as a function of time, of the Jenniskens $D_{\mathrm{J}}$ criterion calculated by comparing the orbits of the Northern $\chi$-Orionids (ORN) and the SPMN061211 fireball with those of NEOs 2002XM35 and 2008XM1.

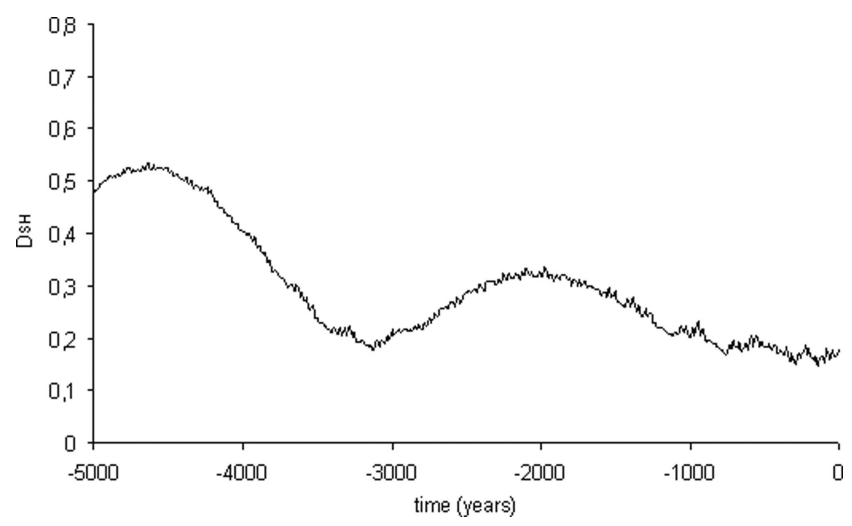

Figure 6. Evolution, as a function of time, of the $D_{\mathrm{SH}}$ criterion calculated by comparing the orbits of asteroids 2002XM35 and 2008XM1.

with the composition inferred for meteor spectroscopy. However, both are very faint (absolute magnitude $(\mathrm{H})$ of 22.96 for 2002 XM35 and 21.93 for 2008XM1), and so obtaining a spectrum will be difficult. From the strength and fireball composition obtained for SPMN061211, we predict that both objects should have primitive petrological signatures associated with carbonaceous chondrites. The identification in the NEO population of primitive asteroids is particularly useful in the context of future sample-return mission (Barucci et al. 2012).

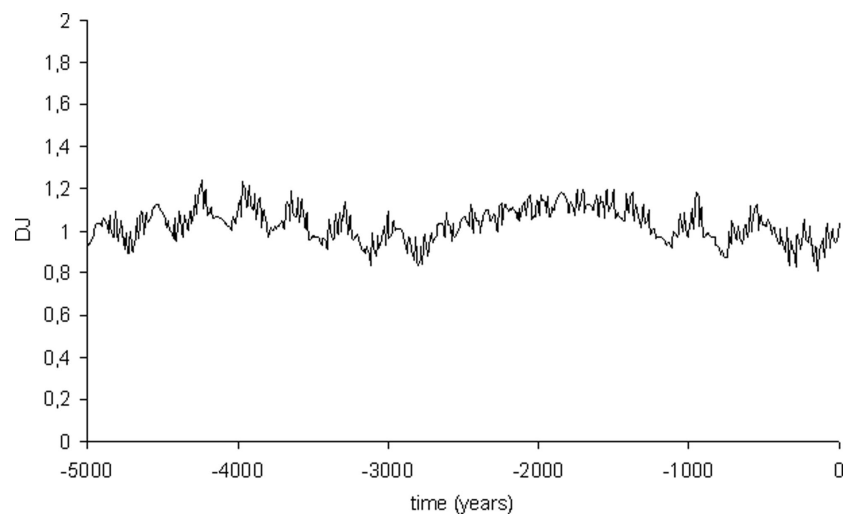

Figure 7. Evolution, as a function of time, of the $D_{\mathrm{J}}$ criterion calculated by comparing the orbits of asteroids 2002XM35 and 2008XM1.

\section{CONCLUSIONS}

We have analysed a mag. -7 double-station Northern $\chi$-Orionid fireball recorded by means of high-sensitivity CCD video devices. On the basis of the orbital elements obtained from this event, we have considered the likely origin of the $\chi$-Orionid meteoroid stream. Our main conclusions are as follows.

(1) The analysis of the SPMN061211 fireball emission spectrum reveals relative chemical abundances compatible with a primitive body. Particularly, the $\mathrm{Mg} / \mathrm{Si}$ ratio seems to be more compatible with a cometary progenitor.

(2) The orbital analysis performed with our orbital association software suggested two potential candidates for parent body of the $\chi$-Orionid meteoroid stream: 2002XM35 and 2008XM1.

(3) The $D_{\mathrm{SH}}$ dissimilarity criterion does not conclusively show which of these two asteroids is a better candidate. However, the criterion proposed by Jenniskens suggests that 2008XM1 is more closely related.

(4) The similar evolution found in the orbits of 2008XM1 and 2002XM35 suggests that they could be fragments or a larger progenitor body. Additional observations to improve the orbital elements of both asteroids, together with highly accurate Northern $\chi$-Orionid orbits, could help us to confirm this.

\section{ACKNOWLEDGEMENTS}

We acknowledge support from the Spanish Ministry of Science and Innovation (projects AYA2009-13227, AYA2009-14000-C0301 and AYA2011-26522), CSIC (grant 201050I043) and Junta de Andalucía (project P09-FQM-4555).

\section{REFERENCES}

Anders E., Grevesse N., 1989, Geochim. Cosmochim. Acta, 53, 197 Babadzhanov P. B., Williams I. P., Kokhirova G. I., 2008, A\&A, 479, 249 Barucci M. A. et al., 2012, Exp. Astron., 33, 645

Ceplecha Z., 1987, Bull. Astron. Inst. Czech., 38, 222

Chambers J. E., 1999, MNRAS, 304, 793

Denning W. F., 1928, J. Br. Astron. Assoc., 38, 302

Drummond J. D., 1981, Icarus, 45, 545

Duprat J. et al., 2010, Sci, 328, 742

Hughes D. W., Williams I. P., Fox K., 1981, MNRAS, 195, 625

Jenniskens P., 1998, Earth Planets Space, 50, 555

Jenniskens P., 2006, Meteor Showers and their Parent Comets. Cambridge Univ. Press, Cambridge

Jenniskens P., 2008, Icarus, 194, 13 
Jopek T. J., 1993, Icarus, 106, 603

Jopek T. J., Rudawska R., Bartczak P., 2008, Earth Moon Planets, 102, 73

Lindblad B. A., 1971a, Smithsonian Contrib. Astrophys., 12, 1

Lindblad B. A., 1971b, Smithsonian Contrib. Astrophys., 12, 14

Madiedo J. M., Trigo-Rodríguez J. M., 2007, Earth Moon Planets, 102, 133

Madiedo J. M., Ortiz J. L., Castro-Tirado A., Díaz M. J., Trigo-Rodríguez J. M., 2010, 41st Lunar and Planetary Science Conference, Woodlands, Texas, p. 1504

Madiedo J. M., Trigo-Rodríguez J. M., Ortiz J. L., Morales N., 2010, Adv. Astron., 1, A 167494

Madiedo J. M., Trigo-Rodríguez J. M., Lyytinen E., 2011a, NASA/CP-2011216469,330

Madiedo J. M., Toscano F. M., Trigo-Rodríguez J. M., 2011b, EPSC-DPS Joint Meeting 2011, Vol. 6, EPSC-DPS2011-72

Madiedo J. M., Trigo-Rodríguez J. M., Ortiz J. L., Castro-Tirado A. J., Pastor S., de los Reyes J. A., 2011c, EPSC-DPS Joint Meeting 2011, Vol. 6, EPSC-DPS2011-73

Nesvorny D., Vokrouhlicky D., 2006, AJ, 132, 1950

Porubčan V., Williams I. P., Kornoš L., 2004, Earth Moon Planets, 95, 697

Porubčan V., Kornoš L., Williams I. P., 2006, Contrib. Astron. Obs. Skalnat'e Pleso, 36, 103

Rietmeijer F., 2000, Meteorit. Planet. Sci., 35, 1025

Rietmeijer F., 2002a, Chemie der Erde, 62, 1
Rietmeijer F., 2002b, Earth Moon Planets, 88, 35

Rudawska R., Vaubaillon J., Atreya P., 2012, A\&A, 541, A2

Schiaparelli G. V., 1867, Astron. Nachr., 68, 331

Southworth R. B., Hawkins G. S., 1963, Smithsonian Contrib. Astrophys., 7,261

Steel D. I., Asher D. J., Clube S. V. M., 1991, MNRAS, 251, 632

Trigo-Rodríguez J. M., 2002, PhD thesis, Universitat de Valencia

Trigo-Rodríguez J. M., Llorca J., 2006, MNRAS, 372, 655

Trigo-Rodríguez J. M., Llorca J., Borovicka J., Fabregat J., 2003, Meteorit. Planet. Sci., 38, 1283

Trigo-Rodríguez J. M. et al., 2007a, MNRAS, 382, 1933

Trigo-Rodríguez J. M., Madiedo J. M., Llorca J., Gural P. S., Pujols P., Tezel T., 2007b, MNRAS, 380, 126

Trigo-Rodríguez J. M., Madiedo J. M., Williams I. P., Castro-Tirado A. J., Llorca J., Vítek S., Jelínek M., 2009, MNRAS, 394, 569

Valsecchi G., Jopek T., Froeschlé C., 1999, MNRAS, 304, 743

Whipple F. L., 1940, Proc. Am. Phil. Soc., 111, 375

Williams I. P., 2011, A\&G, 52, 2.20

This paper has been typeset from a Microsoft Word file prepared by the author. 